\title{
Photoproduction and Electroproduction of Pions in Polarized-Proton Targets.
}

\author{
N. Z AGURY \\ Instituto de Física de la Universidade Católica - Rio de Janeiro \\ A. F. F. TEIXEIRA \\ Centro Brasileiro de Pesquisas Físicas - Rio de Janeiro \\ (Nuovo Cimento, $61 \mathrm{~A}, 83(1969)$ )
}

In the captions of Fig. 4,5,6,8 and 10, instead of "heavy, long-dashed, broken and dashed lines" plase read "solid, broken, dash-dotted and dashed lines".

In the captions of Fig. 7,9 and 11, instead of "dashed, broken and long-dashed lines" please read "broken, dash-dotted and dashed lines".

In the caption of Fig. 12, instead of "heary lines " and "dashed lines " please read, respectively, "solid lines" and "broken lines".

In the caption of Fig. 14, instead of "The long-dashed lines and the lines " please read "The broken lines and the dashed lines". 